\title{
ON A CLASSIFICATION OF THE QUASI YAMABE GRADIENT SOLITONS*
}

\author{
GUANGYUE HUANG ${ }^{\dagger}$ AND HAIZHONG LI $^{\ddagger}$
}

\begin{abstract}
In this paper, we introduce the concept of quasi Yamabe gradient solitons, which generalizes the concept of Yamabe gradient solitons. By using some ideas in $[7,8]$, we prove that $n$ dimensional $(n \geq 3)$ complete quasi Yamabe gradient solitons with vanishing Weyl curvature tensor and positive sectional curvature must be rotationally symmetric. We also prove that any compact quasi Yamabe gradient solitons are of constant scalar curvature.
\end{abstract}

Key words. Locally conformally flat, quasi Yamabe gradient solitons, Weyl curvature tensor.

AMS subject classifications. 53C21, 53C25.

1. Introduction. Let $\left(M^{n}, g\right)$ be an $n$-dimensional Riemannian manifold with $n \geq 3$. If there exists a smooth function $f$ on $M^{n}$ and a constant $\rho$ such that

$$
(R-\rho) g_{i j}=f_{i j},
$$

then we call $\left(M^{n}, g, f\right)$ a Yamabe gradient soliton. Here $R$ denotes the scalar curvature of the metric $g$. If $\rho=0, \rho>0$ or $\rho<0$, then $\left(M^{n}, g, f\right)$ is called a Yamabe steady, Yamabe shrinker or Yamabe expander respectively. Yamabe solitons are special solutions to the Yamabe flow

$$
\frac{\partial}{\partial t} g_{i j}=-R g_{i j}
$$

For the study of the Yamabe flow in the compact case, see $[12,2,1,20,14,19]$ and the references therein. It is very important for understanding the singularity formation in the complete Yamabe flow to study the classification of Yamabe solitons.

In [13], Daskalopoulos and Sesum studied the classification of locally conformally flat Yamabe gradient solitons. They proved

Theorem A. ([13]) If $(M, g, f)$ is a complete locally conformally flat Yamabe gradient soliton satisfying (1.1) with positive sectional curvature, then $(M, g, f)$ is rotationally symmetric.

Theorem B. ([13]) If $(M, g, f)$ is a compact Yamabe gradient soliton, then $g$ is the metric of constant scalar curvature.

In this paper, we consider a generalized Yamabe gradient soliton which we call the quasi Yamabe gradient soliton.

DeFINITION 1.1. If there exists a smooth function $f$ on $M^{n}$ and two constants $m, \rho$ (where $m$ is not zero) such that

$$
(R-\rho) g_{i j}=f_{i j}-\frac{1}{m} f_{i} f_{j}
$$

\footnotetext{
*Received March 12, 2013; accepted for publication June 14, 2013.

${ }^{\dagger}$ Department of Mathematics, Henan Normal University, Xinxiang 453007, P. R. China (hgy@henannu.edu.cn). The research of the first author is supported by NSFC Grant (No. 11001076, 11371018).

${ }^{\ddagger}$ Corresponding author. Department of Mathematical Sciences, Tsinghua University, Beijing 100084, P. R. China (hli@math.tsinghua.edu.cn). The research of the second author is supported by NSFC Grant (No. 11271214).
} 
then we call $\left(M^{n}, g, f\right)$ a quasi Yamabe gradient soliton.

We remark that if $m \rightarrow \infty$, (1.3) reduces to (1.1), so quasi Yamabe gradient solitons can be considered as generalized Yamabe gradient solitons in this sense.

We study classifications for complete quasi Yamabe gradient solitons satisfying (1.3). As in $[7,8]$, the key idea in proving our results is to link the Weyl curvature tensor with the covariant 3 -tensor $D_{i j k}$, introduced by Cao-Chen $[7,6]$, with $D_{i j k}=$ $W_{i j k l} f^{l}$, see Proposition 2.2 in Section 2, where $D_{i j k}$ is defined by

$$
\begin{aligned}
D_{i j k}= & \frac{1}{n-2}\left(R_{k j} f_{i}-R_{k i} f_{j}\right)+\frac{1}{(n-1)(n-2)}\left(R_{i l} g_{j k} f^{l}-R_{j l} g_{i k} f^{l}\right) \\
& -\frac{R}{(n-1)(n-2)}\left(g_{k j} f_{i}-g_{k i} f_{j}\right) .
\end{aligned}
$$

Our main results are as follows:

TheOREM 1.1. Let $(M, g, f)$ be a complete quasi Yamabe gradient soliton satisfying (1.3) with positive sectional curvature.

(1) If $n=3$, then $(M, g, f)$ is rotationally symmetric;

(2) If $n=4$ and $D_{i j k}=0$, then $(M, g, f)$ is rotationally symmetric;

(3) If $n \geq 5$ and $W_{i j k l}=0$, then $(M, g, f)$ is rotationally symmetric.

THEOREM 1.2. If $(M, g, f)$ is a compact quasi Yamabe gradient soliton satisfying (1.3), then $g$ is the metric of constant scalar curvature.

Letting $m \rightarrow \infty$, we have the following result from Theorem 1.1 immediately:

Corollary 1.1. Let $(M, g, f)$ be a complete Yamabe gradient soliton satisfying (1.1) with positive sectional curvature.

(1) If $n=3$, then $(M, g, f)$ is rotationally symmetric;

(2) If $n=4$ and $D_{i j k}=0$, then $(M, g, f)$ is rotationally symmetric;

(3) If $n \geq 5$ and $W_{i j k l}=0$, then $(M, g, f)$ is rotationally symmetric.

REMARK 1.1. Daskalopoulos and Sesum in [13] proved Theorem A (see Theorem 1.3 in [13]) under the assumption that the metric $g$ is locally conformally flat. Theorem A follows from Corollary 1.1. Theorem B follows from Theorem 1.2 when $m \rightarrow \infty$.

REMARK 1.2. Since the 3-tensor $D_{i j k}$ is related to the Weyl curvature tensor by $D_{i j k}=W_{i j k l} f^{l}$ (see Proposition 2.2 in Section 2), we have from Proposition 2.5 in Section 2 that $D_{i j k}=0$ is equivalent to $W_{i j k l} f^{l}=0$ when $n=4$. However, for $n \geq 5$, we can not conclude that $D_{i j k}=0$ implies $W_{i j k l} f^{l}=0$.

REMARK 1.3. For the research of warped product structures of complete gradient Yamabe solitons, see [9]. Some related results for the gradient Ricci solitons can be found in $[18,17,16,11,5,4]$ and the references therein. We note that this paper was posted on Arxiv:1108.6177, on August 31 of 2011.

2. Proof of Theorem 1.1. Throughout this paper, we will agree on the following index convention:

$$
1 \leq i, j, k, \cdots \leq n ; \quad 2 \leq a, b, c, \cdots \leq n .
$$

For convenience, we define

$$
R_{\rho}=R-\rho .
$$


Then (1.3) becomes

$$
R_{\rho} g_{i j}=f_{i j}-\frac{1}{m} f_{i} f_{j}
$$

For quasi Yamabe gradient solitons, we have the following lemma which will be used later:

Lemma 2.1. Let $\left(M^{n}, g, f\right)$ be a quasi Yamabe gradient soliton satisfying (1.3). Then we have

$$
\begin{gathered}
n R_{\rho}=\Delta f-\frac{1}{m}|\nabla f|^{2}, \\
\left(|\nabla f|^{2}\right)_{i}=2 R_{\rho} f_{i}+\frac{2}{m}|\nabla f|^{2} f_{i}, \\
\left(R_{\rho}\right)_{i}=\frac{1}{m} R_{\rho} f_{i}-\frac{1}{n-1} R_{i j} f^{j},
\end{gathered}
$$

where $R_{i j}$ denotes the Ricci curvature of the metric $g$ and $f^{j}=g^{j k} f_{k}$.

Proof. The relationship (2.3) can be obtained directly by contracting the equation (2.2). On the other hand, by choosing the local orthogonal frame $\left\{e_{1}, \cdots, e_{n}\right\}$, we have by use of (2.2),

$$
\left(|\nabla f|^{2}\right)_{i}=2 f_{i j} f_{j}=2\left(R_{\rho} g_{i j}+\frac{1}{m} f_{i} f_{j}\right) f_{j}=2 R_{\rho} f_{i}+\frac{2}{m}|\nabla f|^{2} f_{i} .
$$

Hence, we obtain (2.4).

Using the equation (2.2) again, we obtain

$$
\left(R_{\rho}\right)_{i}=f_{i j j}-\frac{1}{m} f_{i j} f_{j}-\frac{1}{m} f_{i} f_{j j} .
$$

With the help of the Ricci identity, (2.3) and (2.4), we deduce from (2.6)

$$
\begin{aligned}
\left(R_{\rho}\right)_{i} & =f_{i j j}-\frac{1}{m} f_{i j} f_{j}-\frac{1}{m} f_{i} f_{j j} \\
& =(\Delta f)_{i}+R_{i j} f_{j}-\frac{1}{2 m}\left(|\nabla f|^{2}\right)_{i}-\frac{1}{m} f_{i}(\Delta f) \\
& =\left(n R_{\rho}+\frac{1}{m}|\nabla f|^{2}\right)_{i}+R_{i j} f_{j}-\frac{1}{2 m}\left(|\nabla f|^{2}\right)_{i}-\frac{1}{m} f_{i}\left(n R_{\rho}+\frac{1}{m}|\nabla f|^{2}\right) \\
& =n\left(R_{\rho}\right)_{i}+R_{i j} f_{j}+\frac{1}{2 m}\left(|\nabla f|^{2}\right)_{i}-\frac{n}{m} R_{\rho} f_{i}-\frac{1}{m^{2}}|\nabla f|^{2} f_{i} \\
& =n\left(R_{\rho}\right)_{i}+R_{i j} f_{j}+\frac{1}{2 m}\left(2 R_{\rho} f_{i}+\frac{2}{m}|\nabla f|^{2} f_{i}\right)-\frac{n}{m} R_{\rho} f_{i}-\frac{1}{m^{2}}|\nabla f|^{2} f_{i} \\
& =n\left(R_{\rho}\right)_{i}+R_{i j} f_{j}-\frac{n-1}{m} R_{\rho} f_{i},
\end{aligned}
$$

which concludes the proof of (2.5). It completes the proof of Lemma 2.1.

For $n \geq 3$, the Weyl curvature tensor is defined by

$$
\begin{aligned}
W_{i j k l} & =R_{i j k l}-\frac{1}{n-2}\left(R_{i k} g_{j l}-R_{i l} g_{j k}+R_{j l} g_{i k}-R_{j k} g_{i l}\right) \\
& +\frac{R}{(n-1)(n-2)}\left(g_{i k} g_{j l}-g_{i l} g_{j k}\right) .
\end{aligned}
$$


From the definition of the Weyl curvature tensor above, it is easy to see that the Weyl curvature tensor satisfies all the symmetries of the curvature tensor and its traces with the metric are zero. It is well known that $W_{i j k l}=0$ for $n=3$. For $n \geq 4$, $\left(M^{n}, g\right)$ is locally conformally flat if and only if $W_{i j k l}=0$.

As in $[7,6]$, see also $[6,10,8,3]$, we define the following 3 -tensor $D$ by

$$
\begin{aligned}
D_{i j k}= & \frac{1}{n-2}\left(R_{k j} f_{i}-R_{k i} f_{j}\right)+\frac{1}{(n-1)(n-2)}\left(R_{i l} g_{j k} f^{l}-R_{j l} g_{i k} f^{l}\right) \\
& -\frac{R}{(n-1)(n-2)}\left(g_{k j} f_{i}-g_{k i} f_{j}\right) .
\end{aligned}
$$

Then for quasi Yamabe gradient solitons, we have the following consequence:

Proposition 2.2. Let $\left(M^{n}, g, f\right)$ be a quasi Yamabe gradient soliton satisfying (1.3). Then the 3-tensor $D$ is related to the Weyl curvature tensor by

$$
D_{i j k}=W_{i j k l} f^{l} \text {. }
$$

Proof. By use of (2.2) and (2.5), we have

$$
\begin{aligned}
f_{k j i}-f_{k i j} & =\left(R_{\rho} g_{k j}+\frac{1}{m} f_{k} f_{j}\right)_{i}-\left(R_{\rho} g_{k i}+\frac{1}{m} f_{k} f_{i}\right)_{j} \\
& =\left(R_{\rho}\right)_{i} g_{k j}-\left(R_{\rho}\right)_{j} g_{k i}+\frac{1}{m}\left(f_{k i} f_{j}-f_{k j} f_{i}\right) \\
& =\left(R_{\rho}\right)_{i} g_{k j}-\left(R_{\rho}\right)_{j} g_{k i}-\frac{R_{\rho}}{m}\left(g_{k j} f_{i}-g_{k i} f_{j}\right) \\
& =-\frac{1}{n-1}\left(R_{i l} g_{j k} f^{l}-R_{j l} g_{i k} f^{l}\right),
\end{aligned}
$$

where the last equality used (2.5). Using the Ricci identity and (2.7), we have

$$
\begin{aligned}
f_{k j i}-f_{k i j}= & f^{l} R_{l k j i} \\
= & W_{i j k l} f^{l}-\frac{R}{(n-1)(n-2)}\left(g_{i k} g_{j l}-g_{i l} g_{j k}\right) f^{l} \\
& +\frac{1}{n-2}\left(R_{i k} g_{j l}-R_{i l} g_{j k}+R_{j l} g_{i k}-R_{j k} g_{i l}\right) f^{l} \\
= & W_{i j k l} f^{l}+\frac{R}{(n-1)(n-2)}\left(g_{k j} f_{i}-g_{k i} f_{j}\right) \\
& -\frac{1}{n-2}\left(R_{k j} f_{i}-R_{k i} f_{j}\right)-\frac{1}{n-2}\left(R_{i l} g_{j k} f^{l}-R_{j l} g_{i k} f^{l}\right) .
\end{aligned}
$$

Combining (2.10) and (2.11), we obtain by definition (2.8)

$$
\begin{aligned}
W_{i j k l} f^{l}= & \frac{1}{n-2}\left(R_{k j} f_{i}-R_{k i} f_{j}\right)+\frac{1}{(n-1)(n-2)}\left(R_{i l} g_{j k} f^{l}-R_{j l} g_{i k} f^{l}\right) \\
& -\frac{R}{(n-1)(n-2)}\left(g_{k j} f_{i}-g_{k i} f_{j}\right) \\
= & D_{i j k} .
\end{aligned}
$$

Therefore, we complete the proof of Proposition 2.2. 
In particular, by properties of the Weyl curvature tensor and (2.8), we get that $D_{i j k}$ is skew-symmetric in their first two indices and trace-free in any two indices:

$$
D_{i j k}=-D_{j i k}, \quad g^{i j} D_{i j k}=g^{i k} D_{i j k}=0 .
$$

Next, we give a proposition which links the norm of $D_{i j k}$ to the geometry of the level surfaces of the potential function $f$.

Proposition 2.3. Let $\left(M^{n}, g, f\right)$ be a quasi Yamabe gradient soliton satisfying (1.3), and let $\Sigma_{c}=\{x \mid f(x)=c\}$ be the level surface with respect to regular value $c$ of $f$. Then for any local orthonormal frame $\left\{e_{1}, e_{2}, \cdots, e_{n}\right\}$ with $e_{1}=\nabla f /|\nabla f|$ and $\left\{e_{2}, \cdots, e_{n}\right\}$ tangent to $\Sigma_{c}$, we have

$$
\left|D_{i j k}\right|^{2}=\frac{2|\nabla f|^{2}}{(n-1)(n-2)^{2}}\left\{(n-2) R_{1 a}^{2}+(n-1)\left|R_{a b}-\frac{R-R_{11}}{n-1} g_{a b}\right|^{2}\right\},
$$

where $g_{a b}$ is the induced metric on $\Sigma_{c}$.

Proof. From (2.8), we have

$$
\begin{aligned}
\left|D_{i j k}\right|^{2}= & \frac{1}{(n-2)^{2}}\left|R_{k j} f_{i}-R_{k i} f_{j}\right|^{2}+\frac{1}{(n-1)^{2}(n-2)^{2}}\left|R_{i l} g_{j k} f_{l}-R_{j l} g_{i k} f_{l}\right|^{2} \\
& +\frac{R^{2}}{(n-1)^{2}(n-2)^{2}}\left|g_{k j} f_{i}-g_{k i} f_{j}\right|^{2} \\
& +\frac{2}{(n-1)(n-2)^{2}}\left(R_{k j} f_{i}-R_{k i} f_{j}\right)\left(R_{i l} g_{j k} f_{l}-R_{j l} g_{i k} f_{l}\right) \\
& -\frac{2 R}{(n-1)(n-2)^{2}}\left(R_{k j} f_{i}-R_{k i} f_{j}\right)\left(g_{k j} f_{i}-g_{k i} f_{j}\right) \\
& -\frac{2 R}{(n-1)^{2}(n-2)^{2}}\left(R_{i l} g_{j k} f_{l}-R_{j l} g_{i k} f_{l}\right)\left(g_{k j} f_{i}-g_{k i} f_{j}\right) .
\end{aligned}
$$

Let $\left\{e_{1}, e_{2}, \cdots, e_{n}\right\}$ be any local orthonormal frame with $e_{1}=\nabla f /|\nabla f|$ and $\left\{e_{2}, \cdots, e_{n}\right\}$ tangent to $\Sigma_{c}$. That is, under this orthonormal frame, we have $f_{1}=|\nabla f|$ and $f_{2}=f_{3}=\cdots=f_{n}=0$. Thus,

$$
\begin{gathered}
\left|R_{k j} f_{i}-R_{k i} f_{j}\right|^{2}=2|\nabla f|^{2}\left(|R i c|^{2}-R_{1 i}^{2}\right), \\
\left|R_{i l} g_{j k} f_{l}-R_{j l} g_{i k} f_{l}\right|^{2}=2(n-1)|\nabla f|^{2} R_{1 i}^{2}, \\
\left|g_{k j} f_{i}-g_{k i} f_{j}\right|^{2}=2(n-1)|\nabla f|^{2}, \\
\left(R_{k j} f_{i}-R_{k i} f_{j}\right)\left(R_{i l} g_{j k} f_{l}-R_{j l} g_{i k} f_{l}\right)=2|\nabla f|^{2}\left(R R_{11}-R_{1 i}^{2}\right), \\
\left(R_{k j} f_{i}-R_{k i} f_{j}\right)\left(g_{k j} f_{i}-g_{k i} f_{j}\right)=2|\nabla f|^{2}\left(R-R_{11}\right), \\
\left(R_{i l} g_{j k} f_{l}-R_{j l} g_{i k} f_{l}\right)\left(g_{k j} f_{i}-g_{k i} f_{j}\right)=2(n-1)|\nabla f|^{2} R_{11} .
\end{gathered}
$$


Inserting the relationships (2.15)-(2.20) into (2.14) yields $(2.21)$

$$
\begin{aligned}
\left|D_{i j k}\right|^{2}= & \frac{2}{(n-2)^{2}}|\nabla f|^{2}\left(|R i c|^{2}-R_{1 i}^{2}\right)+\frac{2}{(n-1)(n-2)^{2}}|\nabla f|^{2} R_{1 i}^{2} \\
& +\frac{2 R^{2}}{(n-1)(n-2)^{2}}|\nabla f|^{2}+\frac{4}{(n-1)(n-2)^{2}}|\nabla f|^{2}\left(R R_{11}-R_{1 i}^{2}\right) \\
& -\frac{4 R}{(n-1)(n-2)^{2}}|\nabla f|^{2}\left(R-R_{11}\right)-\frac{4 R}{(n-1)(n-2)^{2}}|\nabla f|^{2} R_{11} \\
= & \frac{2|\nabla f|^{2}}{(n-1)(n-2)^{2}}\left\{(n-1)\left(|R i c|^{2}-R_{1 i}^{2}\right)+R_{1 i}^{2}+R^{2}+2\left(R R_{11}-R_{1 i}^{2}\right)\right. \\
& \left.-2 R\left(R-R_{11}\right)-2 R R_{11}\right\} \\
= & \frac{2|\nabla f|^{2}}{(n-1)(n-2)^{2}}\left\{(n-1)|R i c|^{2}-n R_{1 i}^{2}+2 R R_{11}-R^{2}\right\} \\
= & \frac{2|\nabla f|^{2}}{(n-1)(n-2)^{2}}\left\{(n-1)\left(R_{11}^{2}+2 R_{1 a}^{2}+R_{a b}^{2}\right)-n\left(R_{11}^{2}+R_{1 a}^{2}\right)\right. \\
& \left.+2\left(R_{11}+R_{a a}\right) R_{11}-\left(R_{11}^{2}+2 R_{11} R_{a a}+R_{a a} R_{b b}\right)\right\} \\
= & \frac{2|\nabla f|^{2}}{(n-1)(n-2)^{2}}\left\{(n-2) R_{1 a}^{2}+(n-1)\left|R_{a b}-\frac{R_{c c}}{n-1} g_{a b}\right|^{2}\right\} \\
= & \frac{2|\nabla f|^{2}}{(n-1)(n-2)^{2}}\left\{(n-2) R_{1 a}^{2}+(n-1)\left|R_{a b}-\frac{R-R_{11}}{n-1} g_{a b}\right|^{2}\right\} .
\end{aligned}
$$

It completes the proof of Proposition 2.3.

With the help of Proposition 2.3, we can obtain the following results:

Proposition 2.4. Let $\left(M^{n}, g, f\right)$ be a quasi Yamabe gradient soliton satisfying (1.3) with $D_{i j k}=0$, and let $\Sigma_{c}=\{x \mid f(x)=c\}$ be the level surface with respect to regular value $c$ of $f$. Then for any local orthonormal frame $\left\{e_{1}, e_{2}, \cdots, e_{n}\right\}$ with $e_{1}=\nabla f /|\nabla f|$ and $\left\{e_{2}, \cdots, e_{n}\right\}$ tangent to $\Sigma_{c}$, we have

(1) $|\nabla f|^{2}$ and the scalar curvature $R$ of $\left(M^{n}, g_{i j}, f\right)$ are constant on $\Sigma_{c}$;

(2) $R_{1 a}=0$ and $e_{1}=\nabla f /|\nabla f|$ is an eigenvector of $R c$;

(3) the second fundamental form $h_{a b}$ of $\Sigma_{c}$ is of the form $h_{a b}=\frac{H}{n-1} g_{a b}$;

(4) the mean curvature $H=\frac{(n-1) R_{\rho}}{|\nabla f|}$ is constant on $\Sigma_{c}$;

(5) on $\Sigma_{c}$, the Ricci tensor of $\left(M^{n}, g_{i j}, f\right)$ either has a unique eigenvalue $\lambda$, or has two distinct eigenvalues $\lambda$ and $\mu$ of multiplicity 1 and $n-1$ respectively. In either case, $e_{1}=\nabla f /|\nabla f|$ is an eigenvector of $\lambda$. Moreover, both $\lambda$ and $\mu$ are constant on $\Sigma_{c}$.

Proof. Under this chosen orthonormal frame, we have $f_{1}=|\nabla f|$ and $f_{2}=f_{3}=$ $\cdots=f_{n}=0$. When $D_{i j k}=0$, we have from Proposition 2.3 that

$$
R_{1 a}=0
$$

and

$$
R_{a b}=\frac{R-R_{11}}{n-1} g_{a b}
$$

Therefore, we obtain (1) from applying (2.22) to (2.4) and (2.5), respectively. In particular, (2) can be obtained from (2.22) directly. 
By the definition of $h_{a b}$, we have

$$
h_{a b}=\left\langle\nabla_{e_{a}}\left(\frac{\nabla f}{|\nabla f|}\right), e_{b}\right\rangle=\frac{1}{|\nabla f|} f_{a b}=\frac{R_{\rho}}{|\nabla f|} g_{a b},
$$

where the last equality comes from (2.2). Hence, $H=h_{a b} g^{a b}=\frac{(n-1) R_{\rho}}{|\nabla f|}$ is constant from both $R$ and $|\nabla f|$ constant on $\Sigma_{c}$. Thus, (3) and (4) are proved.

Using (2.5), we have

$$
R_{1}=\frac{1}{m} R_{\rho} f_{1}-\frac{1}{n-1} R_{11} f_{1}=|\nabla f|\left(\frac{1}{m} R_{\rho}-\frac{1}{n-1} R_{11}\right)
$$

From the definition of covariant derivative, we have

$$
\begin{aligned}
R_{, 1 a} & =e_{1} e_{a}(R)-\nabla_{e_{a}} e_{1}(R) \\
& =e_{1} e_{a}(R)-\left\langle\nabla_{e_{a}} e_{1}, e_{1}\right\rangle R_{1}-\left\langle\nabla_{e_{a}} e_{1}, e_{b}\right\rangle R_{b} \\
& =e_{1} e_{a}(R) \\
& =0
\end{aligned}
$$

since $R$ is constant on $\Sigma_{c}$. Hence, we obtain from (2.25)

$$
0=R_{, 1 a}=-\frac{|\nabla f|}{n-1} R_{11, a}
$$

Applying

$$
R_{11, a}=e_{a}\left(R_{11}\right)-2 R\left(\nabla_{e_{a}} e_{1}, e_{1}\right)=e_{a}\left(R_{11}\right)-2 h_{a b} R_{1 b}=e_{a}\left(R_{11}\right)
$$

to $(2.27)$ yields

$$
e_{a}\left(R_{11}\right)=0
$$

which shows that $\lambda=R_{11}$ is constant on $\Sigma_{c}$. By means of (2.23) we know that for distinct $a$, the eigenvalues of $R_{a a}$ are the same. Hence, we have the eigenvalue $\mu$ is also constant. This completes the proof of Proposition 2.4.

Proposition 2.5. Let $\left(M^{n}, g, f\right)$ be a quasi Yamabe gradient soliton satisfying (1.3), and let $\Sigma_{c}=\{x \mid f(x)=c\}$ be the level surface with respect to regular value $c$ of $f$.

(1) If $n=3$, then the sectional curvature of $\Sigma_{c}$ with the induced metric is constant;

(2) If $n=4$ and $D_{i j k}=0$, then $W_{i j k l}=0$ on $\Sigma_{c}$. Moreover, the sectional curvature of $\Sigma_{c}$ with the induced metric is constant.

(3) If $n \geq 5$ and $W_{i j k l}=0$, then the sectional curvature of $\Sigma_{c}$ with the induced metric is constant.

Proof. It is well known that, for $n=3$, the Weyl curvature tensor $W_{i j k l}$ vanishes identically. Hence, we obtain $D_{i j k}=0$ from Proposition 2.2. Under the chosen local orthonormal frame as in Proposition 2.4, we have from the Gauss equation and (2.7) and (2.24), for $a \neq b$ :

$$
\begin{aligned}
R_{a b a b}^{\Sigma} & =R_{a b a b}+h_{a a} h_{b b}-h_{a b}^{2} \\
& =R_{a b a b}+\frac{\left(R_{\rho}\right)^{2}}{|\nabla f|^{2}} \\
& =R_{a a}+R_{b b}-\frac{R}{2}+\frac{\left(R_{\rho}\right)^{2}}{|\nabla f|^{2}} \\
& =\frac{R}{2}-R_{11}+\frac{\left(R_{\rho}\right)^{2}}{|\nabla f|^{2}}
\end{aligned}
$$


is constant. It completes the proof of (1).

Next, we prove (2). Since $D_{i j k}=0$, we have from Proposition 2.2

$$
W_{i j k l} f_{l}=0 .
$$

Hence, on the level surface $\Sigma_{c}$, we have

$$
W_{i j k 1}=0, \quad \text { for } 1 \leq i, j, k \leq 4 .
$$

It remains to show that

$$
W_{a b c d}=0, \quad \text { for } 2 \leq a, b, c, d \leq 4 .
$$

This essentially reduces to showing the Weyl curvature tensor is equal to zero in 3 dimensions (see [15], p.276-277 or [7], p.13). Therefore, we have $W_{i j k l}=0$. When $n \geq 4$, the proof for constant sectional curvature is similar. We omit it here. It concludes the proof of Proposition 2.5.

Proof of Theorem 1.1. Following the proof of Daskalopoulos and Sesum in [13], on the level surface $\Sigma_{c}=\{x \mid f(x)=c\}$, we can express the metric $d s^{2}$ as

$$
d s^{2}=\frac{1}{|\nabla f|^{2}}(f, \theta) d f^{2}+g_{a b}(f, \theta) d \theta^{a} d \theta^{b},
$$

where $\theta=\left(\theta^{2}, \cdots, \theta^{n}\right)$ denotes intrinsic coordinates for $\Sigma_{c}$. From (2.29), we know that $\Sigma_{c}$ also has positive sectional curvature with respect to the induced metric. Moreover, Proposition 2.4 and Proposition 2.5 show that $\frac{1}{|\nabla f|^{2}}(f, \theta)=\frac{1}{|\nabla f|^{2}}(f)$ and $g_{a b}(f, \theta)=g_{a b}(f)$. Let $S$ be the set of critical points of $f$. Then the measure of $S$ is zero. Hence, on $M^{n} \backslash S$, we have

$$
d s^{2}=\frac{1}{|\nabla f|^{2}}(f) d f^{2}+g_{a b}(f) d \theta^{a} d \theta^{b}
$$

which shows that $\left(M^{n}, g, f\right)$ is rotationally symmetric by using the arguments as in [13]. We complete the proof of Theorem 1.1.

3. Proof of Theorem 1.2. For any smooth function $u$ on $\left(M^{n}, g\right)$, we introduce the following linear differential operator

$$
L(u)=\operatorname{div}_{m, f}(\nabla u):=e^{\frac{f}{m}} \operatorname{div}\left(e^{-\frac{f}{m}} \nabla u\right) .
$$

Then we have the following:

Lemma 3.1. Let $\left(M^{n}, g\right)$ be a compact Riemannian manifold. Then we have

$$
\int_{M^{n}} v L(u) d \mu=\int_{M^{n}} u L(v) d \mu, \quad \forall u, v \in C^{\infty}\left(M^{n}\right)
$$

where $d \mu$ denotes the measure $e^{-\frac{f}{m}} d V_{g}$. That is, the linear differential operator $L$ is self-adjoint with respect to $L^{2}$ inner product under the measure $d \mu$. In particular, for any smooth function $u$, we have

$$
\int_{M^{n}} L(u) d \mu=0
$$


Proof. A direct calculation gives

$$
\int_{M^{n}} v L(u) d \mu=-\int_{M^{n}} v_{i} u_{i} d \mu=\int_{M^{n}} u L(v) d \mu .
$$

This shows that $L$ is self-adjoint with respect to $L^{2}$ inner product under the measure $d \mu$. (3.3) is a special case when $v=1$ in (3.2). We complete the proof of Lemma 3.1.

Now we come back to prove Theorem 1.2. From (3.1) and (2.3), $L(f)=\Delta f-$ $\frac{1}{m}|\nabla f|^{2}=n R_{\rho}$, thus we have

$$
n \int_{M^{n}} R_{\rho} d \mu=\int_{M^{n}} L(f) d \mu=0
$$

which shows that

$$
\int_{M^{n}} R_{\rho} d \mu=0
$$

By Lemma 2.1, we have by using the second Bianchi identity and by choosing the local orthogonal frame $\left\{e_{1}, \cdots, e_{n}\right\}$,

$$
\begin{aligned}
\Delta\left(R_{\rho}\right) & =\frac{1}{m}\left(R_{\rho}\right)_{i} f_{i}+\frac{1}{m} R_{\rho} f_{i i}-\frac{1}{n-1} R_{i j, i} f_{j}-\frac{1}{n-1} R_{i j} f_{i j} \\
& =\frac{1}{m}\left(R_{\rho}\right)_{i} f_{i}+\frac{1}{m} R_{\rho}\left(n R_{\rho}+\frac{1}{m}|\nabla f|^{2}\right)-\frac{1}{2(n-1)} R_{j} f_{j}-\frac{1}{n-1} R_{i j} f_{i j},
\end{aligned}
$$

which gives

$$
\begin{aligned}
L\left(R_{\rho}\right) & =\Delta\left(R_{\rho}\right)-\frac{1}{m}\left(R_{\rho}\right)_{i} f_{i} \\
& =\frac{n}{m}\left(R_{\rho}\right)^{2}+\frac{1}{m^{2}}|\nabla f|^{2} R_{\rho}-\frac{1}{2(n-1)}\left(R_{\rho}\right)_{i} f_{i}-\frac{1}{n-1} R_{i j} f_{i j} .
\end{aligned}
$$

Applying Lemma 2.1 again, we have

$$
\begin{aligned}
-\frac{1}{n-1} R_{i j} f_{i j} & =-\frac{1}{n-1} R_{i j}\left(R_{\rho} g_{i j}+\frac{1}{m} f_{i} f_{j}\right) \\
& =-\frac{1}{n-1} R_{\rho} R+\frac{1}{m} f_{i}\left[\left(R_{\rho}\right)_{i}-\frac{1}{m} R_{\rho} f_{i}\right] \\
& =-\frac{1}{n-1} R_{\rho} R+\frac{1}{m}\left(R_{\rho}\right)_{i} f_{i}-\frac{1}{m^{2}}|\nabla f|^{2} R_{\rho} .
\end{aligned}
$$

Inserting (3.7) into (3.6), we obtain

$$
L\left(R_{\rho}\right)=\left[\frac{1}{m}-\frac{1}{2(n-1)}\right]\left(R_{\rho}\right)_{i} f_{i}+\frac{n}{m}\left(R_{\rho}\right)^{2}-\frac{1}{n-1} R_{\rho} R .
$$


Integrating (3.8) on $M^{n}$, we obtain by use of (2.1), (3.4) and (3.5)

$$
\begin{aligned}
0 & =\int_{M^{n}} L\left(R_{\rho}\right) d \mu \\
& =\int_{M^{n}}\left\{\left[\frac{1}{m}-\frac{1}{2(n-1)}\right]\left(R_{\rho}\right)_{i} f_{i}+\frac{n}{m}\left(R_{\rho}\right)^{2}-\frac{1}{n-1} R_{\rho} R\right\} d \mu \\
& =\left[\frac{1}{m}-\frac{1}{2(n-1)}\right] \int_{M^{n}}\left(R_{\rho}\right)_{i} f_{i} d \mu+\left[\frac{n}{m}-\frac{1}{n-1}\right] \int_{M^{n}}\left(R_{\rho}\right)^{2} d \mu \\
& =-\left[\frac{1}{m}-\frac{1}{2(n-1)}\right] \int_{M^{n}} R_{\rho} L(f) d \mu+\left[\frac{n}{m}-\frac{1}{n-1}\right] \int_{M^{n}}\left(R_{\rho}\right)^{2} d \mu \\
& =\frac{n-2}{2(n-1)} \int_{M^{n}}\left(R_{\rho}\right)^{2} d \mu,
\end{aligned}
$$

where we used $L(f)=n R_{\rho}$ in the last equality. Clearly, (3.9) shows that $R_{\rho}=0$. Hence, we obtain from $(2.3)$

$$
\Delta f-\frac{1}{m}|\nabla f|^{2}=0
$$

Thus we get that $f$ must be constant since $M^{n}$ is compact. We complete the proof of Theorem 1.2.

\section{REFERENCES}

[1] S. Brendle, Convergence of the Yamabe flow for arbitrary initial energy, J. Differential Geom., 69 (2005), pp. 217-278.

[2] S. Brende, Convergence of the Yamabe flow in dimension 6 and higher, Invent. Math., 170 (2007), pp. 541-576.

[3] S. Brendle, Uniqueness of gradient Ricci solitons, Math. Res. Lett., 18 (2011), pp. 531-538.

[4] H.-D. CAO, Recent progress on Ricci solitons, Recent advances in geometric analysis, pp. 1-38, Adv. Lect. Math. (ALM), 11 Int. Press, Somerville, MA, 2010.

[5] H.-D. CAO, Geometry of complete gradient shrinking Ricci solitons, Geometric and Analysis (Vol I), pp. 227-246, Adv. Lect. Math. (ALM), 17, Int. Press, Somerville, MA, 2011.

[6] H.-D. CAO AND Q. Chen, On locally conformally flat gradient steady solitons, Trans. Amer. Math. Soc., 364 (2012), pp. 2377-2391 .

[7] H.-D. CaO And Q. Chen, On Bach flat gradient shrinking Ricci solitons, Duke Math. J., 162 (2013), pp. 1149-1169.

[8] H.-D. Cao, G. Catino, Q. Chen, C. Mantegazza, and L. Mazzieri, Bach-flat gradient steady Ricci solitons, Calc. Var. Partial Differential Equations, 49 (2014), pp. 125-138.

[9] H.-D. CaO, X. F. Sun, AND Y. Y. Zhang, On the structure of gradient Yamabe solitons, Math. Res. Lett., 19 (2012), pp. 767-774.

[10] Q. Chen And C. X. He, On Bach flat warped product Einstein manifolds, Pacific J. Math., 265 (2013), pp. 313-326.

[11] X. X. Chen AND Y. WANG, On four-dimensional anti-self-dual gradient Ricci solitons, arXiv:1102.0358, 2011.

[12] B. CHOw, The Yamabe flow on locally conformally flat manifolds with positive Ricci curvature, Comm. Pure Appl. Math., 65 (1992), pp. 1003-1014.

[13] P. Daskalopoulos and N. Sesum, The classification of locally conformally flat Yamabe solitons, Adv. Math., 240 (2013), pp. 346-369.

[14] M. DEL Pino AND M. SÁEZ, On the extinction profile for solutions of $u_{t}=\Delta u^{(N-2) /(N+2)}$, Indiana Univ. Math. J., 50 (2001), pp. 611-628.

[15] R. S. Hamilton, Three manifolds with positive Ricci curvature, J. Diff. Geom., 17 (1982), pp. 255-306. 
[16] L. Ni and N. Wallach, On a classification of the gradient shrinking solitons, Math. Res. Lett., 15 (2008), pp. 941-955.

[17] L. Ni AND N. WAllach, On four-dimensional gradient shrinking solitons, Int. Math. Res. Not. IMRN 2008, no. 4, 13pp.

[18] P. Petersen and P. Wylie, On the classification of gradient Ricci solitons, Geom. Topol., 14 (2010), pp. 2277-2300.

[19] H. Schwetlick And M. Struwe, Convergence of the Yamabe flow for "large" energies, J. Reine Angew. Math., 562 (2003), pp. 59-100.

[20] R. YE, Global existence and convergence of Yamabe flow, J. Differential Geom., 39 (1994), pp. 35-50. 
G. HUANG AND H. LI 